\title{
Exprerimental Evaluation of a Dedicated Pinhole SPECT System for Small Animal Imaging and Scintimammography
}

\author{
S. David \\ Department of Medical \\ Instruments Technology \\ Technological Educational \\ Institute of Athens \\ sdavid@teiath.gr
}

\author{
M. Georgiou \\ Department of Medical \\ Instruments Technology \\ Technological Educational \\ Institute of Athens \\ mary_georgiou@yahoo.gr
}

\author{
E. Fysikopoulos \\ Department of Electrical \\ and Computer Engineering \\ National Technical University of \\ Athens \\ lefteris.fysikopoulos@gmail.com
}

\author{
G. Loudos \\ Department of Medical \\ Instruments Technology \\ Technological Educational \\ Institute of Athens \\ gloudos@teiath.gr
}

\begin{abstract}
Nuclear medicine (SPECT and PET) provides functional information, which is complementary to the structural. In cancer imaging radiopharmaceuticals allow visualization of cancer cells functionality, thus small cell population can be imaged. This allows early diagnosis, as well as fast assessment of response to therapy. Our system is a single head gamma camera based on an $\mathrm{R3292}$ position sensitive photomultiplier tube (PSPMT), coupled to a $10 \mathrm{~cm}$ in diameter CsI:Tl crystal. We have assessed two CsI:Tl crystals with pixel size of $2 \times 2 \mathrm{~mm}^{2}$ and $3 \times 3 \mathrm{~mm}^{2}$ respectively. Three collimators were tested: a) a hexagonal, $1.1 \mathrm{~mm}$ in diameter, general purpose parallel hole collimator b) a $1 \mathrm{~mm}$ pinhole and c) a $2 \mathrm{~mm}$ pinhole. Systems were tested using capillary phantoms. All measurements were carried out in photon counting mode with gamma radiation produced by ${ }^{99 \mathrm{~m}}$ Tc. Using the $2 \times 2 \mathrm{~mm}^{2}$ crystal and the $1 \mathrm{~mm}$ pinhole collimator - a resolution better than $1 \mathrm{~mm}$ was achieved. This allows very detailed imaging of small animals. Using the $3 \times 3 \mathrm{~mm}^{2}$ and the $2 \mathrm{~mm}$ pinhole collimator a resolution of $1.3 \mathrm{~mm}$ was possible with suitable sensitivity for breast imaging. Those results indicate that this system is suitable for animal and breast studies. The next step will be clinical evaluation of the camera.
\end{abstract}

Keywords- dedicated gamma camera; PSPMT; small animal imaging; scintimammography; pinhole

\section{INTRODUCTION}

Recently there has been a growing interest in compact and high resolution small gamma cameras, which are used for applications ranging from small animal imaging to scintimammography [1]-[3]. Several groups have been working on the development of the small gamma cameras using position sensitive photomultiplier tubes (PSPMT) and pixellated scintillator crystals [2]-[6]. The spatial resolution of these systems is mainly determined by the degree of pixellation; thus the use of discrete crystals allows the selection of intrinsic spatial resolution of the system independently of scintillator's light yield. This technique assigns an event to a specific crystal location and improves the spatial linearity of the FOV at the edges, compared with a continuous scintillator plate [7], [8].
Image quality in single photon emission computed tomography (SPECT) is strongly influenced by the gamma detector capability to estimate energy and positioning of the $\gamma$ event. Traditionally gamma rays are detected by scintillator crystals that produce a scintillated light pulse, which is amplified, commonly by a position-sensitive photomultiplier tube (PSPMT), and readout via charge division techniques [8, 9]. Detectors based on PSPMTs have the advantages of a continuous photodetector surface (i.e. no gaps between PMTs in Anger camera). Cameras based on PSPMTs are increasingly used for in vivo small animal studies [10], [11].

The benefits of small field of view systems have been explored in clinical environment and mainly in scintimammography [13], [14]. Scintimmamography is a breast imaging technique based on nuclear medicine a technique that involves injection of a radioactive tracer (dye) into the patient. Since the tracer accumulates differently in cancerous and non-cancerous tissues, scintimammography allows physicians to determine whether cancer is present or not. Scintimammography is a supplemental breast exam that is used in specific patient's population, to investigate a breast abnormality, following conventional X-ray mammography and before biopsy.

The past fifteen years there has been increased interest in dedicated breast imagers using Single Photon Emission Mammography (SPEM) or Positron Emission Mammography (PEM) methods; thus a number of such dedicated systems have been developed and some are commercially available. Although X-ray mammography remains the best screening method, it is characterized by low sensitivity, especially in the case of dense breasts. This leads to a large number of negative biopsies, part of which could be avoided. Since, needle biopsy has obvious financial and psychological effects; there is need for a more sensitive second examination. Scintimammography using conventional gamma cameras or PET systems is not sensitive due to the large size of those systems and the limited performance. Typical cases where scintimammography with dedicated breast imagers is clinically useful, is in the case of 
dense breasts or in order to follow up therapy, so that metabolic changes in breast tumors can be depicted a few days following treatment.

In this work, a dedicated single-head round gamma camera based on an R3292 PSPMT, coupled to an $10 \mathrm{~cm}$ in diameter CsI:Tl crystal were tested. Two crystals with pixel size of $2 \times 2 \mathrm{~mm}^{2}$ for small animal imaging and $3 \times 3 \mathrm{~mm}^{2}$ for breast examinations were have assessed, respectively. Performance metrics include a $1 \mathrm{~mm}$ and a $2 \mathrm{~mm}$ pinhole collimator and crystal combinations are compared both for spatial resolution and sensitivity. Images resulting from flooding of the two round CsI:Tl crystals with ${ }^{99 \mathrm{~m}} \mathrm{Tc}$ gamma rays are shown. Moreover profiles of line images from a $1 \mathrm{~mm}$ capillary tube having $50 \mu \mathrm{Ci}$ to $100 \mu \mathrm{Ci}{ }^{99 \mathrm{~m}} \mathrm{Tc}$ were used in order to determine the better full width at half-maximum (FWHM) of the system with respect to the magnification factor of the pinhole apparatus.

\section{MATERIALS AND METHODS}

\section{A. Dedicated gamma ray imager description}

The dedicated small gamma camera consists of a discrete array of $2 \times 2 \times 3 \mathrm{~mm}^{3}$ or a $3 \times 3 \times 5 \mathrm{~mm}^{3} \mathrm{CsI}: \mathrm{Tl}$ crystals with $0.22 \mathrm{~mm}$ spacing coupled to a $12.7 \mathrm{~cm}$ round cross-wire anode PSPMT (Hamamatsu R3292) with simple resistive charge division readout of the $28 \mathrm{x}$ and $28 \mathrm{y}$ cross-wire anodes [15]. The general purpose collimator used has $1.5 \mathrm{~mm}$ diameter and $2.2 \mathrm{~cm}$ long hexagonal holes with $0.2 \mathrm{~mm}$ septa. A resistive current divider network reduced the number of signals from 56 to 4. The four charges arriving at the resistive divider ends (QXa , QXb , QYc and QYd) [16] were preamplified and finally amplified by two 519 of Mech-Tronics Nuclear dual amplifiers (4 channels). The acquisition system is based on a FAST 7070 analog to digital converter (ADC) module connected to a FAST multiparameter acquisition system operating in the Windows environment (MPA/WIN). The multiparameter system controls the four ADCs (dependent mode) and acquires data in list mode through a 1 Mbyte first in - first out register (FIFO) inside the MPA card. The centroid position $(X, Y)$ of the incident light pulse distribution on the photocathode is obtained by Anger's equations. The sum of the four charges provides the total energy deposited by the incident gamma ray. Raw data processing and visualisation was carried out using custom software written in $\mathrm{C}++$. The camera is shielded by $1 \mathrm{~cm}$ lead and by $5 \mathrm{~mm}$ tungsten on the side facing the radiation. A high applied voltage of $950 \mathrm{~V}$ was used; this voltage takes advantage of the full ADCs dynamic range, without noise amplification.

\section{B. Pinhole imaging characteristics and collimator designs.}

The imaging geometry of the pinhole collimator as compared to that of the parallel-hole collimator is shown in Fig.1. The object is positioned close to the pinhole aperture, and a reversed and magnified image is projected onto the detector. Comprehensive discussions of the principle of pinhole imaging in nuclear medicine can be found in [17]. The unique feature of pinhole imaging is that the image is magnified as compared to the image of the parallel-hole collimator. Due to this magnification, the limits imposed by the intrinsic resolution of the camera system can be overcome. In addition, the pinhole collimator also provides a better trade-off between image resolution and photon detection efficiency than the parallel-hole collimator. However, sensitivity is not stable uniform in the entire field of view, thus corrections have to be taken into account.

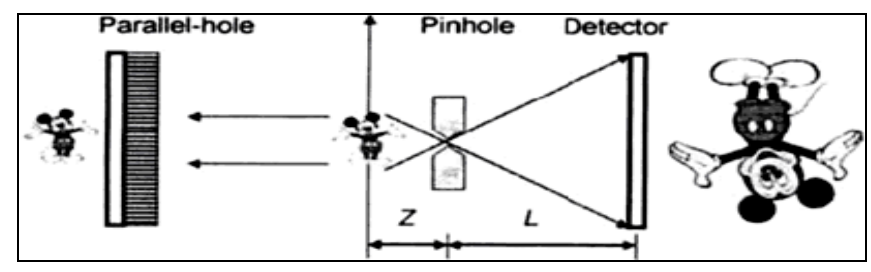

Figure 1. Schematic view of pinhole imaging as compared with parallel-hole collimator. Reproduced by [18]

From the basic pinhole geometry shown in figure $1, \mathrm{Z}$ is the object-to-collimator distance, $\mathrm{L}$ is the collimator length, and a, is the effective hole diameter of the pinhole aperture. The magnification factor $\mathrm{M}$ is given by the equation 1 .

$$
M=L / Z
$$

The resolution of pinhole imaging is determined by the size of the pinhole aperture, the gamma photon energy, and the material used to fabricate the pinhole aperture [18].

\section{Flood correction for parallel collimator.}

Flood correction is a necessary step in pixellized scintillators and is performed following the procedure reported in [19]. The uniformity correction was based on a flood source, consisting of a plastic container filled with radioactive solution of $(2 \mathrm{mCi} 99 \mathrm{mTc})$. The source was sufficiently large to cover the entire detector and was placed in direct contact with the collimated detector. A large number of counts $(4,000,000)$ were collected to minimize the statistical noise. Using the raw flood image - Figure 2(a) - a grid that maps each crystal pixel is determined - Figure 2(b) - and the values in each crystal pixel are summed leading to the flood matrix - Figure 2(c) - that is used to correct raw images.

Figure 3 demonstrates an example of uncorrected image raw image (a), summed image (b) and the flood corrected image (c) for a thin capillary filled with a ${ }^{99 m}$ Tc solution.
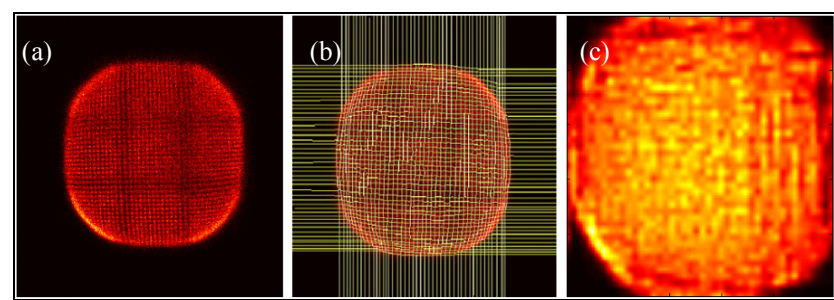

Figure 2. The raw flood image (a); the grid that maps each crystal pixel (b) and the summed flood matrix (c) that is used for flood correction. A flood ${ }^{99 \mathrm{~m}} \mathrm{Tc}$ source has been used. 

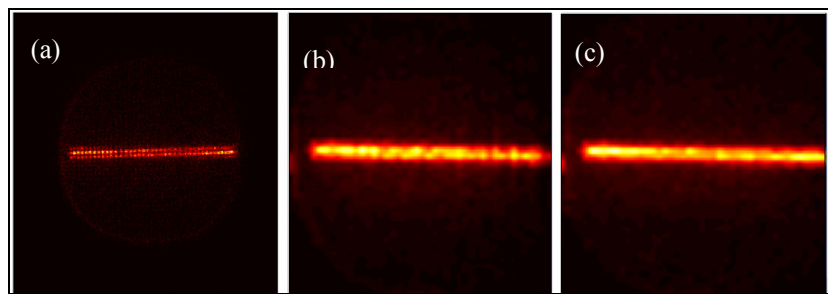

Figure 3. An example of the uncorrected raw image (a), summed image (b) and the flood corrected image (c) of a thin capillary filled with a ${ }^{99 m} \mathrm{Tc}$ solution.

\section{Experimental method}

\section{1) System sensitivity}

The sensitivity was defined as the fraction of events emitted by the line source over those actually detected by the system. We used a $1 \mathrm{~mm}$ capillary tube source filled with a $3.7 \mathrm{Mbq}$ $(100 \mu \mathrm{Ci}){ }^{99 \mathrm{~m}} \mathrm{Tc}$ solution, placed perpendicular to the x-direction across the gamma camera FOV at $0 \mathrm{~mm}$ distance source from collimator. Measurements were carried out for $300 \mathrm{sec}$. Radioactivity decay and corrections in background counts were corrected in sensitivity calculations.

\section{2) System spatial resolution}

Spatial resolution was measured as a function of the distance from source to the detector surface, by using a capillary filled with ${ }^{99 \mathrm{~m} T c}$. Measurements were repeated as capillary stepped (vertically and horizontally) at several positions across the detector FOV. The FWHM of the capillary tubes profiles were calculated using Gaussian fit. FWHM values (in $\mathrm{mm}$ ) were extracted by multiplying by 2.35 times the variance of the distribution $\sigma$. For the evaluation of pinhole apparatus the line source images were obtained. Two different keel edge pinhole collimators with 2 and $3 \mathrm{~mm}$ hole were evaluated. The distance from each pinhole to line source varied horizontally from $1 \mathrm{~mm}$ to $90 \mathrm{~mm}$. The images were acquired using a $100 \%$ energy window. The full width at half-maximum (FWHM) was calculated by the average of three profiles of the line images.

\section{3) System linearity}

Linearity was measured with a flood source phantom consisting of a plastic container filled with ${ }^{99 \mathrm{~m}} \mathrm{Tc}$ radioactive solution with radioactivity varied from $\mu \mathrm{Ci}$ to $22 \mathrm{mCi}$ with increasing activity (in the same volume). The source was placed in direct contact with the collimated detector. A maximum activity in order to have linear response of events was determined.

\section{4) System energy resolution}

System energy resolution is expressed as a percentage and is equal to the photopeak FWHM divided by the photopeak center energy, measured with the collimator in place. System's energy resolution is always larger than the intrinsic. Energy spectra were obtained by placing a ${ }^{99 \mathrm{~m}} \mathrm{Tc}$ point source at the center of the FOV above the detector surface.

All measurements were carried out after the optimization of adjusting parameters (HV, amplifier gain, settings of the discriminators, ADCs coincidence time and conversion gain) that provided maximum rating combined with the best imaging performance.

\section{RESULTS AND DISCUSSION}

Table I shows the results of the measurements regarding, system spatial resolution and system sensitivity of the dedicated gamma camera equipped with $2 \times 2 \times 3 \mathrm{~mm}^{3}$ and $3 \times$ $3 \times 5 \mathrm{~mm}^{3}$ discrete CsI:Tl pixellated crystals at zero source to parallel collimator distance.

TABLE I. GAMMA CAMERA CHARACTERISTICS

\begin{tabular}{|c|c|c|}
\hline Detector characteristics & $\begin{array}{c}3 \times 3 \times 5 \mathrm{~mm}^{3} \\
\text { CsI: } \mathrm{Tl}\end{array}$ & $\begin{array}{c}2 \times 2 \times 3 \mathrm{~mm}^{3} \\
\text { CsI:Tl }\end{array}$ \\
\hline Coating Thickness $\left(\mathrm{g} / \mathrm{cm}^{2}\right)$ & $0.85^{*}$ & $0.68^{*}$ \\
\hline Crystal array pitch & 0.22 & 0.22 \\
\hline $\begin{array}{c}\text { FWHM Spatial Resolution } \\
\text { (mm) }\end{array}$ & 4.5 & 3.7 \\
\hline $\begin{array}{c}\text { FWHM energy resolution } a \\
140 \mathrm{keV}(\%)\end{array}$ & 22 & 23 \\
\hline Sensitivity (Cps / MBq) & 198 & 156 \\
\hline $\begin{array}{c}\text { Quantum Detection Efficiency } \\
@ 140 \mathrm{keV}(\%)\end{array}$ & 85.7 & 68.9 \\
\hline
\end{tabular}

* assuming 100\% packing density

The crystal array with $2 \times 2 \mathrm{~mm}^{2}$ crystal size shows improved detector spatial resolution as compared to the $3 \times 3$ $\mathrm{mm}^{2}$ crystal $(3.7 \mathrm{~mm}$ FWHM and $4.5 \mathrm{~mm}$ FWHM respectively).

Figure 4, shows the spatial resolution variation with increased source to parallel collimator distance.

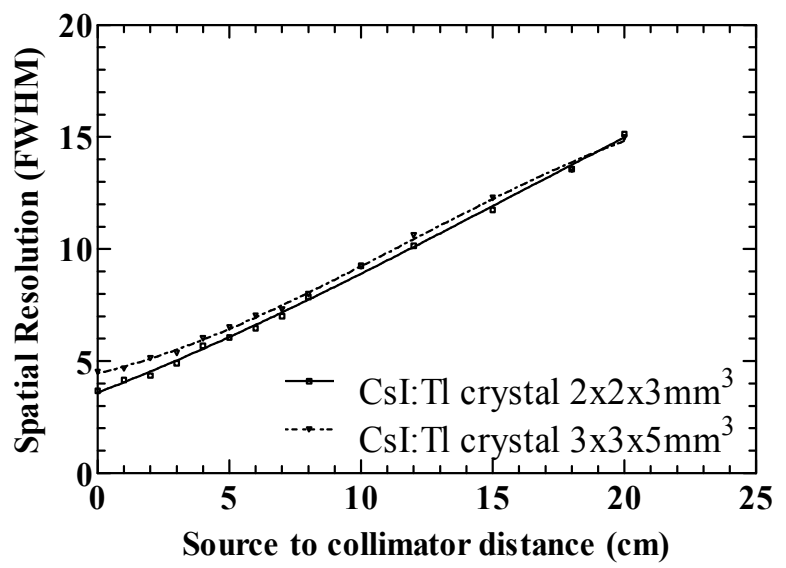

Figure 4. Variation of spatial resolution with source to collimator distance.

The CsI:Tl pixellated scintillator of $2 \times 2 \times 2 \mathrm{~mm}^{3}$ has better resolution properties at distance smaller than $15 \mathrm{~cm}$. Up to this point, spatial resolution properties of pixellated CsI:Tl scintillators is almost the same. The sensitivity of the system remains the same with the variation of source to parallel collimator distance as we expected. 
The imaging geometry of the pinhole collimator as compared to that of the parallel-hole collimator is shown in Figure 3. The unique feature of pinhole imaging is that the image is magnified as compared to the image of the parallelhole collimator. Due to this magnification, the limits imposed by the intrinsic resolution of the camera system can be overcome. However, sensitivity doesn't stable uniform in the entire field of view, thus corrections have to be taken into account. The pinhole to absorption plane distance of $8 \mathrm{~cm}$ secure lower than $50 \%$ efficiency reduction at the edges of FoV. That distance was experimentally determined after positioning a point source at the center of FoV and at the critical angle, varying the pinhole to absorption plane distance. The critical angle is the tapered angle that 'sees' the edge of the detector.

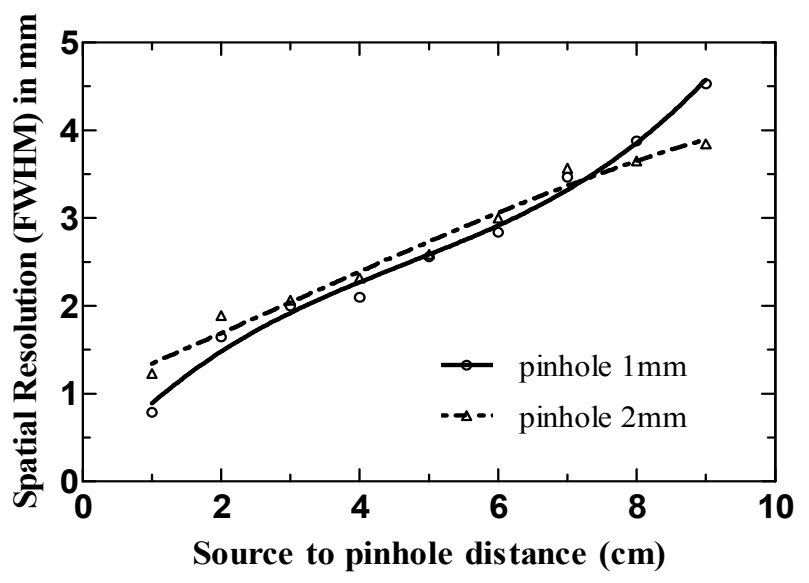

Figure 5. Spatial resolution for the $2 \times 2 \mathrm{~mm}^{2}$ CsI:Tl crystal using the $1 \mathrm{~mm}$ and $2 \mathrm{~mm}$ pinhole collimator.

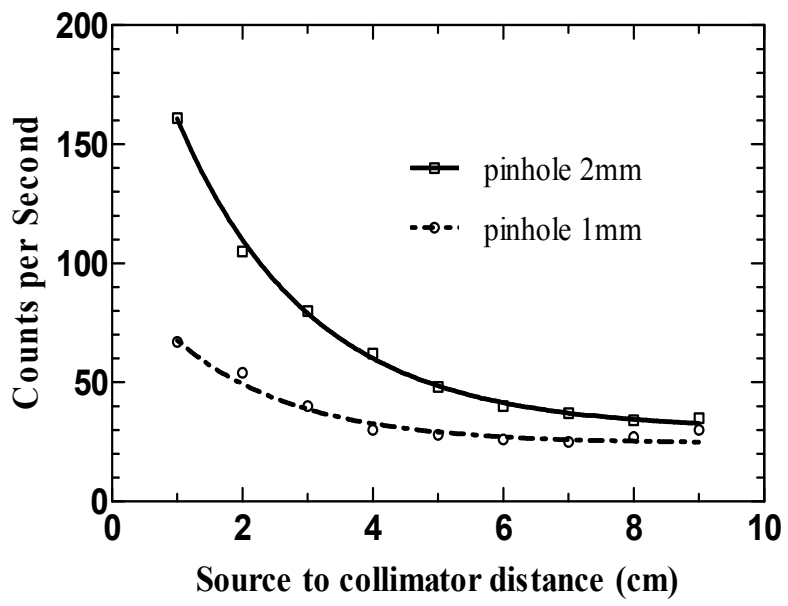

Figure 6: System sensitivity for the $2 \times 2 \mathrm{~mm} 2 \mathrm{CsI}: \mathrm{Tl}$ crystal with 1 and $2 \mathrm{~mm}$ aperture pinhole recorded with increasing source to pinhole distance. A line source with $\sim 50 \mu \mathrm{Ci} 99 \mathrm{mTc}$ radioactivity was imaged; the distance between the crystal surface and the collimator was $8 \mathrm{~cm}$. The source to pinhole distance varied from 1 to 9 .
The spatial resolution as well as the sensitivity recorded by the detector equipped with pinhole collimators is shown in Figures 5 and 6 . The pixellated CsI:TI scintillators were set 8 $\mathrm{cm}$ away from the center of 1 and $2 \mathrm{~mm}$ keel edge pinhole collimators. Metrics were carried out changing the distance of the line source from the collimator. That distance was increased by a step of $1 \mathrm{~cm}$. The measurements were finished when the magnification factor became little down to 1 .

In Figure 7 and 8, the variation of spatial resolution as well as the sensitivity performance achieved by $3 \times 3 \mathrm{~mm}^{2}$ CsI:TI scintillator combined with 1 and $2 \mathrm{~mm}$ keel edge pinhole collimators were shown.

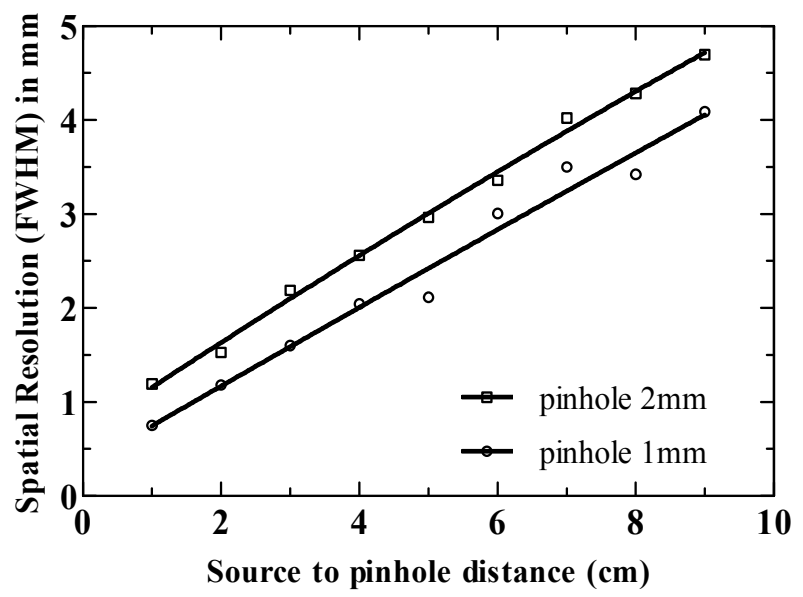

Figure 7: Spatial resolution for the $3 \times 3 \mathrm{~mm}^{2} \mathrm{CsI}: \mathrm{Tl}$ crystal using the $1 \mathrm{~mm}$ and $2 \mathrm{~mm}$ pinhole collimator.

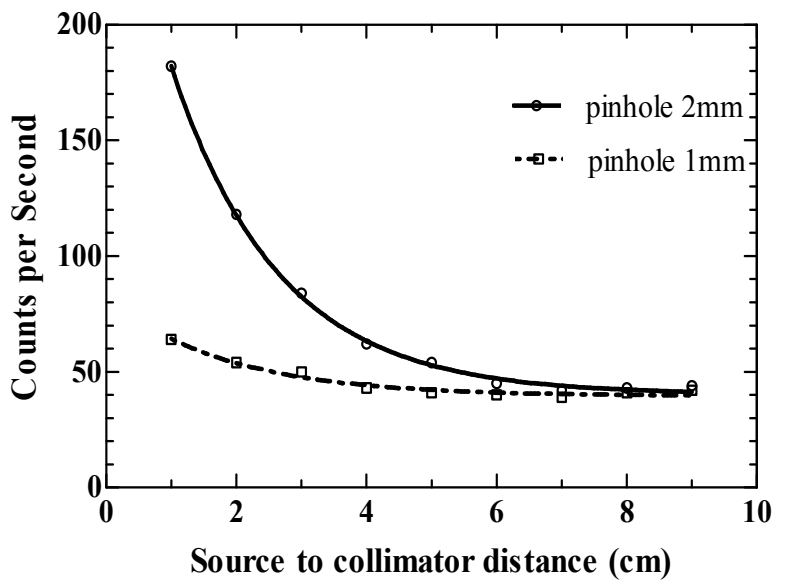

Figure 8: System sensitivity for the $3 \times 3 \mathrm{~mm} 2 \mathrm{CsI}: \mathrm{Tl}$ crystal with 1 and $2 \mathrm{~mm}$ aperture pinhole recorded with increasing source to pinhole distance. A line source with $50 \mu \mathrm{Ci}{ }^{99 \mathrm{~m}} \mathrm{Tc}$ radioactivity was imaged; the distance between the crystal surface and the collimator was $8 \mathrm{~cm}$. The source to pinhole distance varied from 1 to 9 .

For all cases spatial resolution was found best (i.e. 0.79) for: (a) a magnification factor equal to eight (at $1 \mathrm{~cm}$ source to collimator distance), (b) a $1 \mathrm{~mm}$ pinhole aperture, which overcomes the physical limitation of crystal pixel size. The best spatial resolution achieved with the $2 \mathrm{~mm}$ pinhole aperture was 
$1.2-1.3 \mathrm{~mm}$ depending on crystal pixel size. This result is significantly better than that found for the parallel collimator. In all cases, the sensitivity recorded by the system with the pinhole collimator drops exponentially, when source to collimator distance increases. This is in agreement with what is theoretically expected. For distances higher than $8 \mathrm{~cm}$, the sensitivity increases again due to image dimensions decrease.

Point sensitivity and counting rate linearity were evaluated for a range of activities from $\sim 37 \mathrm{MBq}$ to $0.814 \mathrm{GBq}(\sim 1-22$ $\mathrm{mCi}$ ) with a ${ }^{99 \mathrm{~m}} \mathrm{Tc}$ source in air.

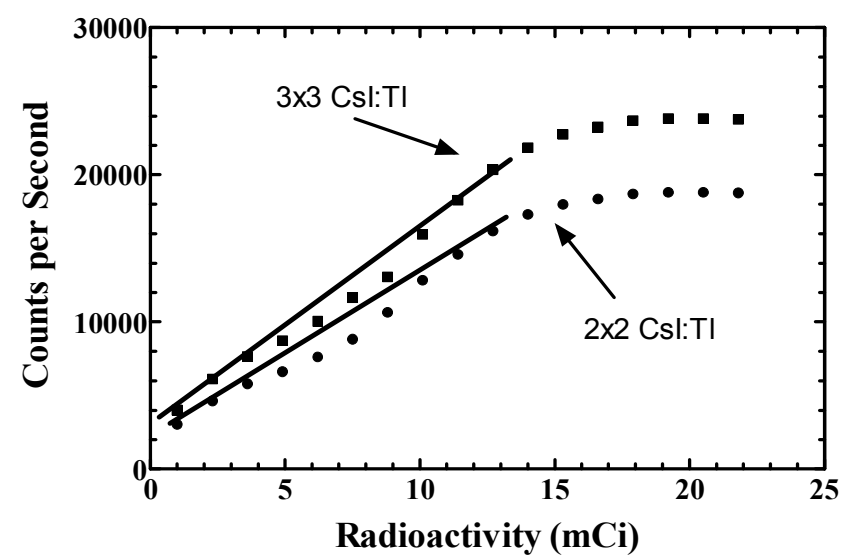

Figure 9: Counting rate linearity for $3 \times 3 \mathrm{~mm}^{2}$ and $2 \times 2 \mathrm{~mm}^{2}$ CsI:Tl pixellated scintillator plates.

A good linearity is evident up to $10 \mathrm{mCi}$ (Figure 9), which is adequate for the activity ranges of small animal imaging and scintimammography. For activity values higher than $10 \mathrm{mCi}$, non-linearities and saturation effects begin to appear. At $20 \mathrm{mCi}$, only a noise signal was imaged. At high count rates, both counts and spatial resolution are lost. The former is due to dead time (the time during which the system cannot process another event), and the latter is primarily due to pulse summation (pile up) of low energy pulses which produce mispositioned signals. After $22 \mathrm{mCi}$ radioactivity, a paralyzing effect drops the counts recorded by the system [20].

\section{CONCUSIONS AND FUTURE WORK}

A dedicated system suitable to perform in vivo imaging of small animals and scintimmamography has been built and tested. Using the $2 \times 2 \mathrm{~mm}^{2}$ crystal and the $1 \mathrm{~mm}$ pinhole collimator a resolution better than $1 \mathrm{~mm}$ was achieved. Using the $3 \times 3 \mathrm{~mm}^{2}$ and the $2 \mathrm{~mm}$ pinhole collimator a resolution of $1.3 \mathrm{~mm}$ was possible with suitable sensitivity for breast imaging. Those results indicate that this system is suitable for animal and breast studies. Future work includes energy spectra correction for each crystal in the array. For each individual crystal spectrum, the location of the photopeak will be identified. Improvement of readout using a subtractive resistive technique will improve raw image quality. Small animal studies are carried out, in order to assess systems performance in dynamic animal studies. The next step is object's rotation, to acquire projection data for pinhole SPECT imaging. Finally breast phantoms are being imaged as an initial step before the clinical evaluation of the camera.

\section{REFERENCES}

[1] R.Pani, M.N. Cinti, R.Pellegrini et al., "Compact large FoV gamma camera for breast molecular imaging", Nucl. Instr. and Meth. A, Vol. 569, pp. 255-259, 2006

[2] Carrie B. Hruska, Michael K. O'Connor and Douglas A. Collins, "Comparison of small field of view gamma camera systems for scintimammography”, Nucl. Med. Commun., Vol. 26, pp. 441-445, 2005

[3] S.Vecchio, N. Belcari, P. Bennati, "A single photon emission computer tomograph for breast cancer imaging", Nucl. Instr. and Meth. A, Vol. 581, pp. 84-87, 2007

[4] R.Wojcik, S. Majewski, D. Steinbach and A.G. Weisenberger, "High Spatial resolution Gamma Imaging Detector Based on 5" Diameter R3292 Hamamatsu PSPMT," IEEE Trans. Nucl. Sci., Vol. 45, No.3, pp.487-491, June, 1998

[5] D.P. McElroy, L.R. MacDonald, F.J. Beekman, Y. Wang, B.E. Patt, J.S Iwanczyk, B.M.W. Tsui, and E.J. Hoffman, "Performance evaluation of A-SPECT: A high-resolution desktop pinhole SPECT system for imaging small animals," IEEE Trans. Nucl. Sci., Vol. 49, No. 5, pp. 2139-2147, 2002

[6] R.Pani, R.Pellegrini, M.N. Cinti et al. , "Imaging detector designs based on flat panel PMT", Nucl. Instr. and Meth. A, Vol. 527, pp. 54-57, 2004.

[7] Del. Guerra, Ionizing Radiation Detectors for Medical Imaging, (Singapore: World Scientific Publishing, Co. Pte. Ltd.), 2004

[8] W. Moses, V. Gayshan, A. Gektin, 'The evolution of SPECT - from Anger to today and beyond' Radiation Detectors for Medical Applications, pp. 37-80, 2006

[9] S. Siegel, R. W. Silverman, Y. Shao, and S. R. Cherry, "Simple charge division readouts for imaging scintillator arrays using a multi-channel PMT" IEEE Trans. Nucl. Sci., Vol. 43, pp. 1634-1641, 1996

[10] V. Popov, S. Majewski, A. G. Weisenberger, and R. Wojcik, "Analog readout system with charge division type output" in IEEE NSS, 2001

[11] D.P.McElroy, et al., "Ultra High resolution in vivo I-125 and Tc-99m small animal pinhole SPECT imaging," in Proc. High Resolution Imaging in Small Animals: Instrumentation, Applications and Animal Handling Conf., Rockville, MD, 2001

[12] R.Pani, et al., "Very high resolution gamma camera based on position sensitive photomultiplier tube," Physica Medica, Vol. 9, No.2-3, pp.233236, 1993

[13] A.Trueman, et al., "Pixellated Csi(Tl) arrays with position-sensitive PMT readout," Nucl. Instrum. Methods A, Vol 353, No.1994, pp. 375378,1994

[14] R.Wojcik, et al., "High spatial resolution gamma imaging detector based on 5" diameter R3292 Hamamatsu PSPMT," IEEE Trans Nucl Sci. Vol. 45, pp. 487-491, 1998

[15] Hamamatsu Corporation, Bridgewater, New Jersey, USA

[16] R. L. Clancy, C. J. Thompson, J. L. Robar, A. M. Bergman “A Simple Technique to Increase the Linearity and Field-of-View in Position Sensitive Photomultiplier Tubes" IEEE Trans. Nucl. Sci., Vol. 44, No. 3, pp. 494-498, 2002, June 1997

[17] Barrett H , Swindell W, (1981) Radiological imaging: The theory of imaging formation, detection, and processing, Vol. 1 \& 2, New York: Academic Press.

[18] QI Yu-Jin., "High-resolution SPECT for small-animal imaging" Nuclear Science and Techniques". Vol.17. No.3, pp. 164-169, 2006

[19] Steinbach D, Majewski S, Williams M, Kross B,Weisenberger A G and Wojcik R, "Development of a small field of view scintimammography camera based on a YAP crystal array and a position sensitive PMT, " IEEE Med. Imag. Conf. Conf. Rec. pp. 1251-1256, 1997

[20] G. F. Knoll, Radiation Detection and Measurement, (Singarore: John Wiley \& Sons), $2^{\text {nd }}$ edition, 1989 


\section{AUTHORS PROFILE}

Stratos David received his Diploma from the Department of Medical Instruments Technology, Technological Educational Institute of Athens, Greece, in 2004. He received his Master Degree in Medical Physics from the Medical School of University of Patras in 2006. He received his $\mathrm{PhD}$ from Medical School of University of Patras in 2010. $\mathrm{He}$ is now working in the field of small field of view detectors for SPECT and PET and detector components evaluation.

Maria Georgiou received her Diploma in electrical and computer engineering from the National Technical University of Athens (NTUA), Greece, in 2007. Since 2009 she is a PhD student in the Medical School of Thessaly. Her work is mainly focused on the development of electronics for field of view SPECT and PET systems and scintimammography.

Eleytherios Fysikopoulos received his Diploma in electrical and computer engineering from the National Technical University of Athens (NTUA), Greece, in 2007. Since 2009 he is a PhD student in National Technical University of Athens. He is working in the field of data acquisition using compact ADCs and FPGAs for application in small SPECT and PET systems.

George Loudos is an Assistant Professor in the Department of Medical Instruments Technology, Technological Educational Institute of Athens, since 2008. He received his Diploma in electrical engineering from the National Technical University of Athens (NTUA), Greece, in 1998 and his $\mathrm{PhD}$ in Biomedical Engineering in 2003 (NTUA). His research interests are focused on Molecular Imaging using Nuclear Medicine Techniques and Medical Instrumentation. More specifically he is interested in the development of dedicated systems for small animal imaging and scintimammography, software for SPECT and PET, Monte Carlo simulations of PET/SPECT systems and radionuclide dosimetry. On application level, he is interested in in vivo imaging of radiopharmaceuticals, radiolebelled nanoparticles and application of SPECT/PET in the study of biological processes. 